\title{
Topological Medial Semigroups
}

\author{
Rushadije R. HALILI *, Merita AZEMI \\ Faculty of Natural Sciences and Mathematics, University of Tetovo, Ilinden n.n., 1200 Tetovo, Republic of \\ North Macedonia
}

*Corresponding Author: Rushadije R. HALILI, Faculty of Natural Sciences and Mathematics, University of Tetovo, Ilinden n.n., 1200 Tetovo, Republic of North Macedonia

\begin{abstract}
In this paper, we prove some results for topological medial semigroups. Throghout, a semigroup will mean a topological semigroup, i.e., a Hausdorff space with a continuous associative multiplication. A medial semigroup is a semigroup satisfaying the medial low.
\end{abstract}

Keywords: Semigroup,medial, topological, homomorphism, quotient topology.

\section{INTRODUCTION}

John B. and Pan, S.J. [2] proved some theorems for topological semigroups. Pettis, B.J.[5] proved some theorems for continuity and openness of homomorphism in topological groups. Paul S., Moster [6] have investigated the structure of topological semigroups. The purpose of this paper is to generalize some of their results to topological medial semigroups.

Let $\mathrm{X}$ and $\mathrm{Y}$ be topological space. Let $p: X \rightarrow Y$ be a surjective (onto) map. The map $p$ is a quotient map provided a subset $\mathrm{U}$ of $\mathrm{Y}$ is open in $\mathrm{Y}$ if and only if $p^{-1}(U)$ is open in $\mathrm{X}$. Let $\mathrm{X}$ and $\mathrm{Y}$ be topological space. Let $p: X \rightarrow Y$ be a surjective (onto) map. Set $C \subseteq X$ is saturated with respect to $p$ if for all $y \in T$ such that $p^{-1}(\{y\}) \cap C \neq \varnothing$ we have $p^{-1}(\{y\}) \subseteq C$. If $C$ saturated with respect to $p$, then for some $A \subseteq Y$ we have $p^{-1}(A)=C$. Let $\mathrm{X}$ and $\mathrm{Y}$ be topological space. Then $p: X \rightarrow Y$ is a quotient map if and only if $p$ is continuoius and maps saturated open sets of $\mathrm{X}$ to open sets of $\mathrm{Y}$. The map $f: X \rightarrow Y$ is an open map if for each open set $U \subseteq X$ the set $f(U)$ is open in Y. If $p: X \rightarrow Y$ is continuous and surjective and $p$ is either open or closed map if for each closed set $A \subseteq X$ the set $f(A)$ is closed in $\mathrm{Y}$. If $\mathrm{X}$ is a space, $\mathrm{A}$ is a set, and $p: X \rightarrow A$ is surjective (onto) map, then there exists exactly one topology $\mathrm{T}$ on $A$ relative to which $p$ is a quotient map. This topology is called the quotient topology induced by $p$.

\section{THEOREMS FOR TOPOLOGICAL HOMOMORPHISM}

A semigroup $S$ is medial if $x a b y=x b a y$ for all $x, a, b, y \in S$. Such a semigroup $S$ satisfies $(x y)^{n}=x^{n} y^{n}$ and $(S x S)^{n}=S^{n} x^{n} S^{n}$ for all $x, y \in S$ and $n \in \mathbb{N}$.

A topological semigroup is a system consisting of a set $S$, an operation " . " and a topology $T$ satisfaying the following conditions:

1) for any $x, y \in S^{\prime}, x y \in S$;

2) for $x, y, z \in S,(x y) z=x(y z)$;

3) the operation "." is continuous in the topology $T$.

A topological subsemigroup $H$ of a semigroup $S$ is a topological subspace of $S$ and also a subsemigroup of $S$.

An equivalence relation $R$ defined on a semigroup $S$ is called homomorphic is for any $a, b, c, d \in S$, $a R b$ and $c R d$ imply $a c R b d$. 
Given an homomorphic equivalence relation $R$ on $S$, we call the set of equivalence classes mod $R$ the quotient set and we denote it by $S / R$.

The mapping from $S$ into $S / R$ defined by $n(x)=$ the class $\bmod R$ to which $x$ belongs is called the natural mapping from $S$ into $S / R$.

The family $U$ of all subsets $U^{*}$ of $S / R$ such that $n^{-1}\left(U^{*}\right)$ is open in $S$ is a topology for $S / R$ and is called quotient topology for $S / R$.

In general use the term homomorphism to mean continuous homomorphism, the terms mapping, function to mean continuous mapping, continuous functions .

Let $S$ be a semigroup, $R$ be a homomorphic equivalence relation on $S$ and let $S / R$ be the quotient set. We define an operation on $S / R$ in the following manner. Suppose that $A$ and $B$ are two arbitrary elements in $S / R$, then $A B=C$ if for any $a \in A$ and $b \in B$ we have $a b \in C$. This operation is welldefined because $R$ is a homomorphic equivalence relation. Also it is associative, because the semigroup $S$ is associative.Therefore the quotient set $S / R$ with the operation just defined is a semigroup. We call it the quotient semigroup.

We say a semigroup $S$ statisfies the condition $A$ if for every open set $U$ of $S$, the subset $n^{-1}(n(U))$ is also open where $n$ is the natural mapping from $S$ onto $S / R$.

Theorem1: If the medial semigroup $S$ statisfies the condition $A$, then the quotient set $S / R$ is a topological medial semigroup with the quotient topology, and the natural mapping $n$ from $S$ into $S / R$ is an open topological homomorphism.

Proof: We have shown that $S / R$ is an abstract semigroup. Now we wish to show that the natural mapping $n$ from $S$ to $S / R$ is an abstract homomorphism.

Let $X$ and $Y$ be two equivalence classes $\bmod R$ and let $X Y=Z$. Then by definition of the operation in $S / R$, for any $x \in X$ and $y \in Y, x y \in Z$. Since the natural mapping $n$ assigns each element to the class it belongs, we have $n(X)=X, n(Y)=Y$, and $n(x y)=n(z)=Z$. These equations together with the equation $X Y=Z$ imply that $n(x y)=n(x) n(y)$.

This shows that the natural mapping $n$ is an abstract homomorphism from $S$ into $S / R$.

Now let $U^{*}$ be an open set in $S / R$. By the definition of the quotient topology for $S / R, n^{-1}\left(U^{*}\right)$ is open. Hence $n$ is continuous. Let $U$ be an open set in $S$. Since $S$ satisfies the condition $A$, $n^{-1}[n(U)]$ is open. Then by definition of the quotient topology, $n(U)$ is open.

Now we wish to show that the semigroup operation in $S / R$ is continuous. Let $A$ and $B$ be two orbitrary elements in $S / R$ such that $A B=C$. Suppose that $W^{*}$ is an open neighborhood of $C$. Then $W=n^{-1}\left(W^{*}\right)$ is an open neighborhood of $C$, considered as a subset of $S$. Since the semigroup operation in $S$ is continuous, for every $a \in A$ and every $b \in B$ such that $a b=c$, there is an open neighborhood $U_{a}$ of $a$ and an open neighborhood $V_{b}$ of $b$ such that $U_{a} V_{b} \subset W$. Choose such a neighborhood $V_{b}$ for every $b \in B$. Then $\underset{\substack{a \in A \\ b \in B}}{U_{a} V_{b}}=\left[\bigcup_{a \in A} U_{a}\right]\left[\bigcup_{b \in B} V_{b}\right] \subset W$.

Now $U_{a \in A} U_{a}$ is an open neighborhood of $A$ in $S$, and $n$ is an open mapping. It follows that $n\left[U_{a \in A} U_{a}\right]$ is an open neighbordood of the element $A$ in $S / R$. Similarly $n\left[U_{b \in B} V_{b}\right]$ is an open neighborhood of the element $B$ in $S / R$. Since $\left[U_{a \in A} U_{a}\right]\left[U_{b \in B} V_{b}\right] \subset W$, we have

$$
n\left[\bigcup_{a \in A} U_{a}\right] n\left[U_{b \in B} V_{b}\right]=n\left[U_{a \in A} U_{a} \underset{b \in B}{U} V_{b}\right] \subset n(W)=W^{*}
$$


Hence we have found an open neighborhood $n\left[\bigcup_{a \in A} U_{A}\right]$ of $A$ and an open neighborhood $n\left[{ }_{b \in B} V_{b}\right]$ of $B$ such that $n\left[U_{a \in A} U_{a}\right] n\left[{ }_{b \in B} V_{b}\right] \subset W^{*}$. This shows that the semigroup operation in $S / R$ is continuous.

Theorem2: If $S$ and $T$ are two medial semigroups and $g$ is a homomorphism from $S$ into $T$ then $g$ induces a homomorphic equivalence relation $R_{g}$ on $S$.

Proof: We define a relation $R_{g}$ on $S$ in the following manner. Suppose that $a$ and $a^{*}$ are two element: of $S$, then : $a=a^{*}$ if and only if $g(a)=g\left(a^{*}\right)$. Evidently, $R_{g}$ is an equivalence relation.

We show that $R_{g}$ is homomorphic, i.e., if $a, a^{*}, b, b^{*} \in S$ such that $a=a^{*} \bmod R_{g}$ and $b=b^{*} \bmod R_{g}$, then $a b=a^{*} b^{*} \bmod R_{g}$. Now $a=a^{*} \bmod R_{g}$ implies $g(a)=g\left(a^{*}\right)$ and $b=b^{*} \bmod R_{g}$ implies $g(b)=g\left(b^{*}\right)$. These two equations imply that $g(a) g(b)=g\left(a^{*}\right) g\left(b^{*}\right)$.

Since $g$ is a homomorphism we have $g(a) g(b)=g(a b)$ and $g\left(a^{*}\right) g\left(b^{*}\right)=g\left(a^{*} b^{*}\right)$.

Hence $g(a b)=g\left(a * b^{*}\right)$.

Theorem3: Let $S$ and $T$ be two topological medial semigroups and let $g$ be an open homomorphism from $S$ onto $T$. Then

a) $S / R_{g}$ is a topological medial semigroup with the quotient topology;

b) the natural mapping $n$ from $S$ onto $S / R_{g}$ is an open homomorphism;

c) the mapping $h$ from $S / R_{g}$ onto $T$ defined by $h(A)=g(a)$ for any $a \in A$ as a subset of $S$ and $A \in S / R_{g}$ is a topological isomorphism.

Proof: By theorem2, $g$ includes a homomorphic equivalence relation $R_{g}$ on $S$. Let $S / R_{g}$ be the quotient set. Then $S / R_{g}$ is a medial semigroup. Let $n$ be the natural mapping from $S$ onto $S / R_{g}$. We show that the medial semigroup $S$ satisfies the condition $A$.

Let $U$ be an open subset in $S$. Since $g$ is an open map, $g(U)$ is open in $T$. Also $g$ is continuous. Hence the subset $g^{-1}[g(U)]$ is open in $S$. But $g^{-1}[g(U)]=\{x \in s / g(x)=g(y)$ for some $y \in U\}$ and $n^{-1}[n(U)]=\{x \in S / g(x)=g(y)$ for some $y \in U\}$ thence $n^{-1}[n(U)]=g^{-1}[g(U)]$ and $n^{-1}[n(U)]$ is open. This shows that $S$ satisfies the condition $A$. Since $S$ satisfies the condition $A$, the parts a) and b) follow from theorem1. Before proving part c), we wish to show that the mapping $h$ defined in the theorem is well-defined.

Let $A$ be any element of $S / R_{g}$ and let $a^{*}$ and $a^{* *}$ be any two elements of $A$ as a subset of $S$. Then $a^{*}=a^{* *} \bmod R_{g}$. This implies $g\left(a^{*}\right)=g\left(a^{* *}\right)$. Hence $h(A)=g\left(a^{*}\right)=g\left(a^{* *}\right)$.

This shows that $h$ is well-defined. Also $h$ is a one to one mapping.

For each $A \in S / R_{g}$ there corresponds a unique value $h(A)=g(a)$ in $T$ as shown above. 
Now since $g$ is a mapping from $S$ onto $T$, for each $t \in T$ there is an element $a \in S$ such that $t=g(a)$, by definition of $R g, a=b \bmod R_{g}$ if and only if $g(a)=g(b)$.

It follows that for each $g(a)=t$, there is one and only one equivalence class $A \bmod R_{g}$ such that $h(A)=g(a)=t$. Hence $h$ is a one to one mapping. We further show that $h$ is an algebraic homomorphism. Let $A$ and $B$ be any two elements in $S / R_{g}$. Then $h(A B)=g(a b)=g(a) g(b)=h(A) h(B)$, where $a$ and $b$ are orbitrary elements of $A$ and $B$ respectively. This shows that $h$ is an algebraic homomorphism.

We show also that $h$ is continuous. Let $A$ be an element in $S / R_{g}$ such that $h(A)=t$ and let $W$ be an open neighborhood of $t$.

Since $h(A)=g(a)$ for every $a \in A$, and since $g$ is continuous, for every $a \in A$, there is an open neighborhood $U_{a}$ of $a$ such that $g\left(U_{a}\right) \subset W$.

Choose such an open neighborhood $U_{a}$ for every $a \in A$. Then $\bigcup_{a \in A}\left(U_{a}\right)$ is a neighborhood of $A$ in $S$ and $n\left[{ }_{a \in A}\left(U_{a}\right)\right]$ is an open neighborhood of the element $A$ in $S / R_{g}$. But

$g\left[\underset{a \in A}{U}\left(U_{a}\right)\right]=h\left\{n\left[U_{a \in A}^{U}\left(U_{a}\right)\right]\right\} \subset W$.

So for any neighborhood $w$ of $h(A)$, we have found a neighborhood $n\left[\underset{a \in A}{U}\left(U_{a}\right)\right]$ of $A$ such that $h\left\{n\left[\underset{a \in A}{U}\left(U_{a}\right)\right]\right\} \subset W$. This shows that $h$ is continuous.

Finally we show that $h$ is open. Let $U^{*}$ be an open subset of $S / R_{g}$. Since the natural mapping $n$ from $S$ onto $S / R_{g}$ is continuous, $n^{-1}\left(U^{*}\right)$ is an open subset in $S$ into $T$. So $g\left[n^{-1}\left(U^{*}\right)\right]$ is open on $T$.

But $g\left[n^{-1}\left(U^{*}\right)\right]=h\left\{n\left[n^{-1}\left(U^{*}\right)\right]\right\}=h\left(U^{*}\right)$. Hence $h\left(U^{*}\right)$ is open in $T$. This shows that $h$ is an open mapping. This completes the proof.

If the medial semigroup $S$ satisfies the condition $A$, then the quotient set $S / R$ is a topological medial semigroup with the quotient topology, and the natural mapping $n$ from $S$ onto $S / R$ is an open topological homomorphism.

Conversely, if $g$ is an open homomorphism from $S$ into a medial semigroup $T$, then $T$ is topologically isomorphic to the quotient semigroup $S / R_{g}$, where $R_{g}$ is homomorphic equivalence relation defined by $a R_{g} b$ if and only if $g(a)=g(b), a, b \in S$.

\section{Fundamental Theorem of Homomorphism of the Topological Medial Semigroups}

Theorem4: Let $S$ and $T$ be two topological medial semigroups both satisfying the condition $A$.

Let $g$ be an open homomorphism from $S$ onto $T$ and let $R *$ be a homomorphic equivalence relation defined on $T$. Then there is a homomorphic equivalence relation $R$ on $S$ and there is a mapping $h$ from $S / R$ onto $T / R^{*}$ which is a topological isomorphism.

Proof: Since $R^{*}$ is a homomorphic equivalence relation on $T$, by theorem $1, T / R^{*}$ is a topological medial semigroup and the natural mapping $n$ from $T$ onto $T / R^{*}$ is an open topological homomorphism. Since the mapping $g$ from $T$ onto $T / R^{*}$ is an open topological homomorphism.

Since the mapping $g$ from $S$ onto $T$ is also a homomorphism, it follows that the product mapping $n g$ from $S$ onto $T / R^{*}$ is also a homomorphism. We show that $n g$ is open. 
Let $U$ be an open set in $S$. Since $g$ is open $g(U)$ is open in $T$. Also, $n$ is an open map;

So $n g(U)$ is open in $T / R^{*}$. This shows that $n g$ is an open topological homomorphism.

Now $S$ and $T / R^{*}$ are two topological medial semigroups. $S$ satisfies the condition $A$, and $n g$ is an open topological homomorphism from $S$ onto $T / R^{*}$. Hence, by theorem $2 n g$ induces a homomorphic equivalence relation $R_{n g}$ and $T / R^{*}$. Denote $R_{n g}$ by $R$. Then we have $S / R \cong T / R^{*}$.

We call this isomorphism $h$.

\section{REFERENCES}

[1] Clifford, A. H., and Preston, G. B., The Algebraic Theory of Semigroups I, Math Surveys, 7, Amer. Math. 50., 1961.

[2] John B., Pan, S.J. Topological semigroups, Gazeta de Matematica (19-24).

[3] Balci Dervis, Zur theorie der topologichen n-grupen, Minerva publication-Munich 1981.

[4] G.Crombez, G.Six, On topological n-groups, Abhand . Math. Semin. Miv. Hamburg 41 (1974), 115-124.

[5] Pettis, B.J., On continuity and openness of homomorphism in topological groups, Anals of Mathematics-LII (1950) 293-308.

[6] Paul S. Mostert, The structure of topological semigroups.

[7] [7]J. L. Chrislok, On medial semigroups, Journal of Algebra 12, 1-9(1969).

Citation: Rushadije R. HALILI \& Merita AZEMI, Topological Medial Semigroups, International Journal of Scientific and Innovative Mathematical Research (IJSIMR), vol. 8, no. 10, pp. 18-22, 2020. Available : DOI: https://doi.org/ 10.20431/2347-3142.0810003

Copyright: (C) 2020 Authors. This is an open-access article distributed under the terms of the Creative Commons Attribution License, which permits unrestricted use, distribution, and reproduction in any medium, provided the original author and source are credited. 